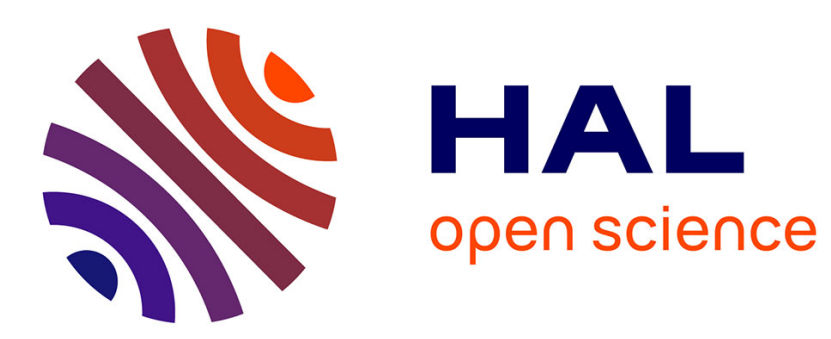

\title{
Efficient prototyping of large-scale pdms and silicon nanofluidic devices using pdms-based phase-shift lithography
}

Yannick Viero, Qihao He, Laurent Mazenq, Hubert Ranchon, Jean-Yves Fourniols, Aurélien Bancaud

\section{To cite this version:}

Yannick Viero, Qihao He, Laurent Mazenq, Hubert Ranchon, Jean-Yves Fourniols, et al.. Efficient prototyping of large-scale pdms and silicon nanofluidic devices using pdms-based phase-shift lithography. Microfluidics and Nanofluidics, 2012, 12 (1-4), pp.465 - 473. 10.1007/s10404-011-0888-0 . hal-01682518

\section{HAL Id: hal-01682518 \\ https://hal.science/hal-01682518}

Submitted on 15 Jan 2018

HAL is a multi-disciplinary open access archive for the deposit and dissemination of scientific research documents, whether they are published or not. The documents may come from teaching and research institutions in France or abroad, or from public or private research centers.
L'archive ouverte pluridisciplinaire HAL, est destinée au dépôt et à la diffusion de documents scientifiques de niveau recherche, publiés ou non, émanant des établissements d'enseignement et de recherche français ou étrangers, des laboratoires publics ou privés. 


\title{
EFFICIENT PROTOTYPING OF LARGE-SCALE PDMS AND SILICON NANOFLUIDIC DEVICES USING PDMS-BASED PHASE SHIFT LITHOGRAPHY
}

\author{
Y. Viero ${ }^{1,2, *}$, Q. He ${ }^{1,2, *}$, L. Mazenq ${ }^{1,2}$, H. Ranchon ${ }^{1,2}$, J.Y. Fourniols ${ }^{1,2}$, A. Bancaud ${ }^{1,2}$ \\ ${ }^{1}$ CNRS ; LAAS ; 7 avenue du colonel Roche, F-31077 Toulouse Cedex 4, France \\ ${ }^{2}$ Université de Toulouse ; UPS, INSA, INP, ISAE ; UT1, UTM, LAAS ; F-31077 Toulouse \\ Cedex 4, France \\ * these authors equally contributed to this work
}

Correspondance: abancaud@laas.fr

\begin{abstract}
In this study, we explore the potential of Poly-DiMethylSiloxane (PDMS)-based phase shift lithography (PPSL) for the fabrication of nanofluidic devices. We establish that this technology, which was already shown to allow for the generation of $100 \mathrm{~nm}$ linear or punctual features over $\mathrm{cm}^{2}$ surfaces with conventional photolithography systems, is readily adequate to produce some of the most popular nanofluidic systems, namely nanochannels and nanoposts arrays. We also demonstrate that PPSL technology enables to generate PDMS and silicon nanofluidic systems. This technological achievement allows us to perform single DNA molecule manipulation experiments in PDMS and silicon nanochannels, and we observe an unexpectedly slow migration of DNA in PDMS devices, which is independent on salt or pH conditions. Our data in fact hint to the existence of an anomalous response of DNA in PDMS nanofluidic devices, which is likely associated to transient non-specific interactions of DNA with PDMS walls. Overall, our work demonstrates the efficiency and the performances of PPSL for prototyping nanofluidic systems.
\end{abstract}




\section{Introduction}

In the last decade, a large number of functionalities have been miniaturized in microfluidic systems in order to perform complex chemical or biochemical procedures with enormous benefits in cost, reduced sample volumes, and improved performances (Sanders et Manz, 2000; Reyes et al., 2002). The ambition to downscale microfluidic operations in nanoscale devices has led to the emergence of nanofluidics, which consists in performing analytical operations with systems composed of structures of $\sim 100 \mathrm{~nm}$ or less (Mawatari et al., 2010; Piruska et al., 2010). Nanofluidic systems offer a unique potential in analytical sciences because separation operations can be performed in matrices tailored at the nanoscale, enabling to achieve some of the most impressive separation efficiencies (Kaji et al., 2004). Moreover, the confined geometry of nanofluidic systems allows to monitor the environment of single molecules, and the conformation of single DNA molecules was successfully manipulated inside nanochannels (Reisner et al., 2005; Tegenfeldt et al., 2004).

The rapid breakthrough of microfluidics largely relied on the huge fabrication toolbox that was invented for microsystems processing, but the development of nanofluidics has been hampered by the difficulty of fabricating nanostructures due to the cost and the poor accessibility of nanofabrication equipments (Whitesides, 2011). For example, electron beam lithography (EBL) is the most commonly used nanofabrication technology (Fontana et al., 2002), as it achieves exquisite spatial resolutions, but it is a slow process because nanopatterns are imprinted by point-by-point serial exposures. Moreover, the typical write field for an e-beam of $1 \mathrm{~mm}^{2}$ is relatively small for a separation device (Dorfman, 2010), and larger write-fields are possible using a step-and-repeat method, albeit the possibility of stitching errors. NanoImprint Lithography (NIL), which has been developed in the middle of the 90s and has been shown to be adapted to fabricate $25 \mathrm{~nm}$ features (Chou et al., 1995), is one popular alternative to EBL. Although clearly relevant to mass production, NIL is not ideal for prototyping given the need to fabricate a mold by e-beam patterning. Consequently, there is a clear need for alternative methods for prototyping nanofluidic systems, as exemplified by the recent report on nanopost arrays fabrication based on conventional photolithography ( $\mathrm{Ou}$ et al., 2011).

In this article, we demonstrate that PDMS-based Phase Shift Lithography (PPSL) is a useful technology for a broad range of applications in nanofluidics. PPSL, which was initially pioneered by Whitesides and colleagues in 1997 (Rogers et al., 1997), relies on a PDMS phase mask that is obtained by conventional photolithography. By exposing a photoresist through this mask, $100 \mathrm{~nm}$ linear or punctual features have been generated, and optimal performances with $50 \mathrm{~nm}$ patterns were achieved using hard-PDMS (h-PDMS, a formulated PDMS with a 5-fold increased Young modulus (Schmid et Michel, 2000)). This technology was already applied to generate optical polarizers (Rogers et al., 1997), nanowells to accommodate individual $\mathrm{NaCl}$ crystals (Odom et al., 2002), or high-frequency surface acoustic wave transducers (Hesjedal and Seidel, 2003). The relevance of PPSL for nanofluidic devices fabrication yet remains to be demonstrated, and we provide different solutions for the integration of $\sim 100 \mathrm{~nm}$ PPSL features in functional nanofluidic systems made out of silicon or PDMS. Single DNA manipulation experiments are subsequently conducted in our 
nanochannels and nanoposts arrays with exquisite signal to noise ratio at the single molecule level. The electrophoretic response of DNA in PDMS vs. silicon nanochannels is specifically investigated, showing an unexpectedly slow mobility in PDMS likely associated to non specific interaction between PDMS and DNA. Overall, we posit that PPSL offers a great technological solution for nanofluidics, and it is particularly relevant to overcome the technological bottleneck, which has slowed down innovation rate in nanofluidics.

\section{Phase-Shift Photolithography: general principles}

\subsection{Principle}

PPSL aims to fabricate nanometric patterns with conventional photolithography. It relies on a transparent PDMS phase mask, which is composed of periodically distributed surface air gaps (Fig. 1a). As light passes through this mask, its intensity is modulated in the near field due to the phase shifting at the PDMS/air regions (Rogers et al., 1998). This phase shift can be modulated by adjusting the thickness of the patterns imprinted on the mask according to:

$$
\Delta \varphi=\frac{2 \pi}{\lambda} h \times\left(n_{P D M S}-n_{\text {air }}\right)
$$

with $\lambda, h$, and $n$ the wavelength, the channel height, and the optical index, respectively. The intensity in the near field is reduced to zero as the phase shift is equal to an odd multiple of $\pi$. Thus, given the difference in optical index between PDMS and air of 0.33, and the peak absorption wavelength of a photoresist of $\sim 350 \mathrm{~nm}$, the light intensity at the edges of the gaps is reduced to zero for $h \sim 500 \mathrm{~nm}$. The light cancellation spans over a width on the order of one quarter of the wavelength of the light used for exposure, so that we expect to produce $\sim 90 \mathrm{~nm}$ features with this technology.

\subsection{Application to positive and negative tone photoresists}

The master for the binary phase mask was fabricated by conventional photolithography of AZ1505 photoresist (AZ-Electronic Materials), and it consisted of periodic $4 \mu \mathrm{m}$ linear patterns repeated every $8 \mu \mathrm{m}$ (Fig. 1A). Note that we coated silicon wafers with an HexaMethylDiSiloxane (HMDS) layer by vapor priming in order to promote adhesion of AZ1505. Unreticulated PDMS (Sylgard 184, Corning) was poured on these structures, and cured at $75{ }^{\circ} \mathrm{C}$ during 3 hours. The PDMS template was then peeled off, and manually brought into conformal contact with a silicon wafer coated with AZ1505 (prebake $30 \mathrm{~s}$ at $95^{\circ} \mathrm{C}$, red layer in Fig. $1 \mathrm{~A}$ ). The photoresist with the phase shift mask was exposed with $75 \mathrm{~mJ} / \mathrm{cm}^{2}$ (h-line) using a conventional SUSS Microtech ${ }^{\circledR}$ Ma6 mask aligner, and subsequently developed during $20 \mathrm{~s}$ with AZ developer. Linear patterns of $200 \mathrm{~nm}$ were obtained with this protocol (Fig. 1B, left panel), and smaller motifs of $\sim 100 \mathrm{~nm}$ could be generated by reducing the prebake time to $20 \mathrm{~s}$ (Fig. 1B, right panel). Indeed a shorter (or colder) prebake is responsible for a higher concentration in residual solvent in the photoresist. As a result, the development rate increases, leading to a reduction in size of the patterns. Notably, these structures were imprinted over large surfaces of several $\mathrm{cm}^{2}$, so that one key 
benefit of PPSL stems for its capacity to generate very long features characterized by a length-to-width ratio of $\sim 10^{4}$ (5 mm / $200 \mathrm{~nm}$ ).

PPSL was also shown to allow for the fabrication of 2D arrays of nanoposts by means of two successive exposures along different directions (Rogers et al., 1997). Each exposure indeed imprints one array of parallel lines on the photoresist, and the intersections between these lines define punctual unexposed features. Interestingly, by choosing the angular shift between the exposures and the dose of illumination from $30-50 \mathrm{~mJ} / \mathrm{cm}^{2}$ with the Ma6 mask aligner, the size and the geometry of the posts could be tuned down to sizes of $120 \mathrm{~nm}$ (Fig. 1B). Note that the exact dose of illumination had to be calibrated every month in order to obtain optimal results. In addition to being low-cost and high-throughput, the features produced with this technology were highly reproducible, as demonstrated by the standard deviation in size of less than 3\% for $270 \mathrm{~nm}$ nanoposts, as inferred from structural inspection of individual nanoposts by electron microscopy (Supplementary Fig. S1).

We finally developed a protocol for PPSL of the negative tone photoresist SU8-2000.5 (Microchem Corp), which was spin coated at $4000 \mathrm{rpm}$ during $30 \mathrm{~s}$ with a closed bowl to reach a minimal thickness of $90 \mathrm{~nm}$ (blue layer in Fig. $1 \mathrm{~A}$ ). After a prebake of $60 \mathrm{~s}$ at $95^{\circ} \mathrm{C}$, the photoresist was exposed to $40 \mathrm{~mJ} / \mathrm{cm}^{2}$ (i-line) with an EVG® 620 mask aligner, postbaked during $30 \mathrm{~s}$ at $95^{\circ} \mathrm{C}$, and eventually developed during $1 \mathrm{~min}$ in Propylene Glycol Methyl Ether Acetate (PGMEA). This protocol allowed us to obtain $250 \mathrm{~nm}$ linear patterns, which could not be further reduced most likely because the post-baking step favors diffusion of reticulation agents, and thus broadens the resulting patterns, as described in (Odom et al., 2002).

Overall our results are similar to those published by Whitesides and colleagues, and we set out to devise fully-integrated nanofluidic systems with PPSL.

\section{Integration of PPSL features in nanofluidic devices}

Nanofluidic devices are composed of macroscopic inlets/outlets, of microscopic fluidic connections that enable to convey molecules, and of functional nanoscale features. Their fabrication is thus based on multi-scale hierarchical processes that we describe in the following.

\subsection{Transfer and connection of PPSL features}

Let us first consider the simple case of nanopost arrays. PPSL patterns were transferred in silicon using standard Reactive Ion Etching (RIE) with an STS or an Alcatel system with $\mathrm{CF}_{4}, \mathrm{SF}_{6}$ and $\mathrm{O}_{2}$ pressures at $1.310^{-3} \mathrm{mBar}$ or $7.210^{-2} \mathrm{mBar}$ and RF powers set to $450 \mathrm{~W}$ or $2800 \mathrm{~W}$, respectively. Etch rates were $9 \mathrm{~nm} / \mathrm{s}$ and $22 \mathrm{~nm} / \mathrm{s}$ for the STS and the Alcatel, respectively, as inferred from mechanical profilometry using a TENCOR profiler. Access holes were finally drilled through silicon by sand blasting using a $50 \mu \mathrm{m}$ film of Laminar 5038 to protect micro and nanopatterns (Fig. 2A).

\subsection{Fabrication of silicon nanochannels}


Transferred PPSL nanochannels were connected to microfluidic channels, which were generated by conventional photolithography. Note that micro- and nano-channels were systematically connected because the arrays of nanochannels covered areas of several $\mathrm{cm}^{2}$, and the typical distance between microchannels was much lower of $\sim 500 \mu \mathrm{m}$ (cartoon in Fig. 2B). The microchannels were etched by RIE over depths of $\sim 2 \mu \mathrm{m}$ (see inset of Fig. $2 \mathrm{~B}$ for dimensions), and access holes were eventually drilled through silicon by sand blasting.

A thermal oxide layer of $\sim 100 \mathrm{~nm}$ was grown on silicon for electrical insulation in the case of devices dedicated to DNA electrophoresis (Jeong et al., 2001). This additional oxide layer reduces the width of nanochannels by $100 \mathrm{~nm}$ while keeping the depth unchanged.

\subsection{Fabrication of a silicon master for PDMS molding}

The generation of a master for PDMS molding required the development of a specific two-step process, starting with the etching of microchannels by RIE followed by the fabrication of nanochannels by PPSL (Fig. 2C). PPSL was thus performed on a textured substrate, which was not optimal for photoresist spin-coating, and for PDMS phase mask conformal contact. We indeed obtained poorly-resolved PPSL features at the interface between micro- and nano-structures for $2 \mu \mathrm{m}$ thick microchannels (Supplementary Fig. S2), and this artifact was significantly improved using $700 \mathrm{~nm}$ thick microchannels (Fig. 2C). Note that the width of microchannels was carefully adjusted to $30 \mu \mathrm{m}$ in order to prevent the collapse of PDMS chip after molding (Zhou et al., 2005).

The resulting mold was immersed in trichloroethylene supplemented with $1 \%$ octadecyl-trichlorosilane during 5 minutes in order to obtain anti-fouling surfaces. Unreticulated h-PDMS was then spin coated on the mold at $1000 \mathrm{rpm}$ during $30 \mathrm{~s}$ to produce a $40 \mu \mathrm{m}$ layer that was cured at $75{ }^{\circ} \mathrm{C}$ during 45 minutes. $30 \mathrm{~g}$ of conventional PDMS prepolymer was subsequently poured on the wafer, and the baking was prolonged during 2 hours at $75{ }^{\circ} \mathrm{C}$. The resulting double-layer PDMS replica, which is coined h-PDMS chip thereafter, was peeled off, and holes were punched at the end of the each microchannels using a Harris uni-core punch kit. Note that the apparition of crackles on the PDMS surface was minimized by punching 24 hours after peeling. The quality of nanochannels replication was eventually assayed by electron microscopy (Fig. 2D).

\subsection{Sealing nanofluidic structures}

After thorough cleaning of glass coverslips and h-PDMS chips with 95\% saturated chromium trioxide mixture and isopropanol, respectively, glass and h-PDMS surfaces were activated using oxygen plasma during 2 minutes at $800 \mathrm{~W}$, and $30 \mathrm{~s}$ at $200 \mathrm{~W}$, respectively, before being placed in conformal contact. The bonding strength was enhanced by curing the resulting chip at $100^{\circ} \mathrm{C}$ during 20 minutes (Tang et al., 2006).

In the case of silicon devices, the standard sealing technique is anodic bonding, which involves large electric fields in combination with strong pressures. These conditions however turned out to be deleterious for nanoposts stability (not shown). Bonding was thus performed by spin-coating a $6 \mu \mathrm{m}$ layer of h-PDMS on glass coverslips (4000 rpm), which was cured at 
$75^{\circ} \mathrm{C}$ for 2 hours, and then activated using the same protocol as for h-PDMS chip bonding. We checked by optical microscopy that nanopillars remained intact after this process (not shown). Interestingly, because the PDMS layer is dissolved in 95\% saturated chromium trioxide mixture at room temperature during a few hours, silicon nanofluidic chips could be repeatedly cleaned and re-assembled for each new experiment.

\section{Application to single DNA molecule manipulation}

\subsection{Methods}

We wished to conduct single DNA molecule manipulation experiments with our nanofluidic devices. $\lambda$-DNA molecules fluorescently labeled with YOYO-1 (Molecular Probes) were prepared after careful titration of both species by absorbance spectroscopy at $260 \mathrm{~nm}$ and $488 \mathrm{~nm}$. Two buffers, namely Tris-Borate-EDTA (TBE) (Tris-HCl $80 \mathrm{mM}$, Borate $80 \mathrm{mM}$, Ethylenediaminetetraacetic acid (EDTA) $5 \mathrm{mM}$ ) or $100 \mathrm{mM}$ phosphate buffers, were used at various dilutions and they were supplemented with 5\% Dithiothreitol and 2.5\% Poly- vinylpyrrolydone (PVP, MW $40 \mathrm{kDa}$ ) to reduce photo-induced damages, and to suppress non specific interactions and electro-osmotic flows, respectively. Note that the addition of higher concentration of PVP increases the buffer viscosity to a degree that prevents the complete filling of nanochannels, thus impeding the electric field to pass across the device.

Imaging was performed with a Zeiss epifluorescence microscope equipped with the 38HE filter set (Zeiss), and with a Lumencor Light Engine emitting at $475 \mathrm{~nm}$ with a $28 \mathrm{~nm}$ bandwidth and a power of $20 \mathrm{~mW}$ to reduce photobleaching (Akerman, 1996). An ANDOR iXon-885 camera was used to observe single DNAs using a binning of $4 \times 4$, and a pixel size of $0.33 \mu \mathrm{m}$. Exposure times varied from $10 \mathrm{~ms}$ to $30 \mathrm{~ms}$ depending on the requirements of the experiment. DNA manipulation was performed with pressure or electrophoretic actuation using a Fluigent ${ }^{\circledR}$ pressure manager in the range 5 to $1000 \mathrm{mBar}$, or a GW INSTEK® power supply (1-100 V) connected to platinum electrodes directly immersed in the fluidic reservoirs, respectively.

\subsection{Single molecule observations}

We first checked that PPSL nanopost arrays were adapted to study the dynamics of DNA-nanopost interaction. The physics of the collision of $\lambda$-DNA with obstacles has been extensively worked out at the micron-scale, and more recently with smaller posts of $\sim 500 \mathrm{~nm}$ (Kaji et al., 2004; Ou et al., 2011). Yet the subject remains sparsely studied for smaller sized cylindrical or elliptical obstacles. Using $200 \mathrm{~nm}$ cylindrical posts, we could investigate DNA unhooking dynamics at the single molecule level with exquisite temporal and spatial resolutions, as demonstrated by the time lapse of one individual collision event, and the time evolution of the molecule center of mass (Fig. 3A). In addition large numbers of molecules could be simultaneously observed with no detectable problems of clogging (inset in Fig. 3A), thus showing that our technology is compatible with systematic investigations of DNAnanoposts interactions (Y.V. \& A.B., in preparation). 
The functionality of h-PDMS and silicon nanochannels was subsequently assessed by performing experiments with $\lambda$-DNA, which could be manipulated in $200 \mathrm{~nm}$ square nanochannels with lengths spanning $100 \mu \mathrm{m}$ to $5 \mathrm{~mm}$ (Fig. 3B). The confinement inside nanochannels expectedly induced an entropic spreading of the molecules to a length of $\sim 4 \mu \mathrm{m}$ (plateau in Fig. 3B and histogram in inset), representing a degree of stretching of $28 \%$ given that fluorescently labeled $\lambda$-DNA measures $\sim 22 \mu \mathrm{m}$ (Perkins et al., 1995). This value is consistent with experimental results obtained with glass nanochannels (Reisner et al., 2005). Interestingly, we could perform DNA nanomanipulation experiments during several hours with our h-PDMS chips by continuously applying $10 \mathrm{mBar}$ in both microchannels. Moreover, high quality imaging and parallel manipulations of DNA could be performed with h-PDMS nanochannels, as shown by the time lapse sequence of one uptake event, and by the fluorescence micrograph displaying several molecules entering multiple nanochannels, respectively (Fig. 3B). Consequently, our technology for nanochannel and nanopost arrays fabrication is particularly adapted for nanofluidic prototyping.

\subsection{Characterization of DNA electrophoresis in h-PDMS chips}

PDMS is the workhorse material in microfluidics due its facile and rapid fabrication by replication molding (Becker and Gärtner, 2007). However PDMS has been sparsely used in nanofluidics because its Young's modulus is too low to prevent nanostructures from collapsing (Zhou et al., 2005), although in some cases PDMS collapse was cleverly directed to generate nanostructures of tunable dimensions (Huh et al., 2007; Park et al., 2009). Using our technology for $200 \mathrm{nmx} 200 \mathrm{~nm}$ PDMS nanochannels fabrication (Fig. 2D), we set out to evaluate the performances of this material for the electrophoretic manipulation of DNA.

The mobility of $\lambda$-DNA, which was assessed by measuring the velocity of the center of mass of at least 5 molecules under at least 4 different tensions, was $2-3 \times 10^{-4} \mathrm{~cm}^{2} / \mathrm{Vs}$ in $2 \mu \mathrm{m}$ thick access microchannels for different dilutions of TBE (Fig. 4A-B). This value was in excellent agreement with previous estimates obtained in microfluidic systems (see e.g. (Ou et al., 2009); (Randall and Doyle, 2006)). The migration of DNA was also characterized in nanochannels, showing a 10-fold slowed down mobility that was independent of salt concentration and pH (Fig. 4B). Moreover, the comparison of the mobility of DNA fragments of different sizes, which spontaneously form due to light-induced DNA fragmentation (note the sizes of the DNA fragments in Fig. 3B), did not indicate any appreciable difference (data not shown). Interestingly, this slow migration of DNA in PDMS nanochannels was already detected in another study that reported a mobility of $\sim 0.6 \mathrm{~cm}^{2} / \mathrm{Vs}$ (Campbell et al., 2004). This result is somewhat surprising because DNA is generally treated as a free draining polymer moving in an electric field at a rate that solely depends on its effective charge density, and not on its conformation (Viovy, 2000). One may therefore hypothesized that electro-osmotic flows are enhanced in nanochannels, leading to a reduced effective migration speed in comparison to the free mobility of DNA of $4.5 \times 10^{-4} \mathrm{~cm}^{2} / \mathrm{Vs}$ in TBE (Stellwagen et al., 1997). However, electro-osmotic flows should rather be reduced in nanochannels because the Debye length is not necessary negligible in comparison to nanochannels half width, so that the electro-osmotic flow does not reach a flat profile, and the maximum fluid velocity is lowered. 
It may also be tempting to suggest that the roughness of PDMS nanochannels sidewalls enhances electro-osmotic flows, but recent simulations rather demonstrated the opposite trend (Qiao, 2006). Altogether, explanations that rely on changes in electrophoresis or electroosmosis thus appear to be inconsistent, and the measurement of a rapid DNA mobility of $1.9 \times 10^{-4} \mathrm{~cm}^{2} /$ Vs (Mannion et al., 2006) and $1.1 \times 10^{-4} \mathrm{~cm}^{2} /$ Vs (Fig. 4A) in $100 \mathrm{nmx} 100 \mathrm{~nm}$ glass nanochannels or in our own silicon $200 \mathrm{nmx} 200 \mathrm{~nm}$ nanochannels, respectively, further strengthens this argument. Notably, the mobility in our devices is somewhat smaller than that in glass nanochannels, most likely because our sealing layer is composed of h-PDMS.

One possible explanation may be that the DNA is becoming entangled in the PVP, which is the surface coating polymer. PVP should indeed be present in higher concentration in nanochannels due to their increased surface-to-volume ratio, yet this scenario is unlikely because the molecular weight of the PVP is relatively low, and it is inconsistent with the higher mobility in silicon nanochannels. We then investigated whether the altered DNA migration in nanochannels was induced by the existence of permeation flows inward PDMS (Randall and Doyle, 2006). But the mobility remained unchanged after super saturating our devices in humid conditions. PDMS is also known to contain residues such as unreticulated chains or platinum ions that degrade imaging conditions (Jo et al., 2007), and which can be eliminated by soaking PDMS in EDTA during 24h. Notably, this protocol was performed by immersing nanofluidic chips in EDTA after the final bonding step, because, in our hands, the EDTA treatment was incompatible with the covalent sealing of the devices. To our surprise, DNA mobility was 2-fold enhanced in microchannels and 10-fold slowed down in nanochannels, thus showing that PDMS plays a central role in this unexpectedly slow mobility. We therefore speculated that the anomalous mobility of DNA was associated to the existence of transient non-specific interactions between PDMS and DNA. Interestingly we assayed the electrophoretic response of $100 \mathrm{~nm}$ carboxyl-nanoparticles, i.e. a nano-object with a different surface chemistry, in untreated PDMS and with 0.5X TBE. These objects were characterized by a mobility of $1.6 \mathrm{~cm}^{2} / \mathrm{Vs}$ and $0.03 \mathrm{~cm}^{2} / \mathrm{Vs}$ in microchannels and nanochannels, respectively. Thus the migration of these nano-objects is sharply slowed down in nanochannels, generalizing the idea that the migration of nano-objects in h-PDMS nanochannels exhibits anomalous behaviors. Consequently, our data suggest h-PDMS offers an attractive solution for rapid nanofluidic prototyping, but this material may also introduce uncontrolled bias for quantitative physical studies.

\section{Conclusions}

In this work we demonstrate that PPSL allows to generate some of the most popular structures of nanofluidics, namely nanochannels and nano-posts arrays. Moreover, PPSL can be specifically adapted to fabricate silicon and PDMS nanofluidic systems. Beyond this technological achievement, we show that high quality single DNA molecule imaging can be performed with our PPSL systems. Finally, we perform a series of experiments to analyze the anomalous electrophoretic response of DNA in PDMS nanochannels, which is characterized by a 10-fold slowed down mobility. This study leads us to conclude that PDMS is particularly relevant for nanofluidic prototyping, but this material may introduce uncontrolled biases for 
physical studies. Because PPSL solely requires conventional photolithography equipments, we envision that PPSL can be an efficient solution to speed up the discovery rate in nanofluidics.

Acknowledgments Y.V. and H.R. thank DGA and for PhD fellowship funding, and we thank TEAM research facility for support in fabrication. This work and the fellowship of Q.H. were supported by the ANR program JC08_341867.

\section{Bibliography}

AAkerman, B., et Tuite, E. (1996). Single-and double-strand photocleavage of DNA by YO, YOYO and TOTO. Nucleic acids research 24, 1080.

Becker, H., et Gärtner, C. (2007). Polymer microfabrication technologies for microfluidic systems. Anal Bioanal Chem 390, 89-111.

Campbell, L. C., Wilkinson, M. J., Manz, A., Camilleri, P., et Humphreys, C. J. (2004). Electrophoretic manipulation of single DNA molecules in nanofabricated capillariesElectronic supplementary information (ESI) available: Four videoclips showing the movement of DNA molecules in nanocapillaries. See http://www.rsc.org/suppdata/lc/b3/b312592k/. Lab Chip 4, 225.

Chou, S. Y., Krauss, P. R., et Renstrom, P. J. (1995). Imprint of sub-25 nm vias and trenches in polymers. Appl. Phys. Lett. 67, 3114.

Dorfman, K. (2010). DNA electrophoresis in microfabricated devices. Rev. Mod. Phys. 82, 2903-2947.

Fontana, R. E., Katine, J., Rooks, M., Viswanathan, R., Lille, J., MacDonald, S., Kratschmer, E., Tsang, C., Nguyen, S., Robertson, N., et al. (2002). E-beam writing: a nextgeneration lithography approach for thin-film head critical features. Magnetics, IEEE Transactions on 38, 95-100.

Hesjedal, T., et Seidel, W. (2003). Near-field elastomeric mask photolithography fabrication of high-frequency surface acoustic wave transducers. Nanotechnology 14, 91.

Huh, D., Mills, K. L., Zhu, X., Burns, M. A., Thouless, M. D., et Takayama, S. (2007). Tuneable elastomeric nanochannels for nanofluidic manipulation. Nat Mater 6, 424428.

Jeong, Y., Kim, S., Chun, K., Chang, J., et Chung, D. S. (2001). Methodology for miniaturized CE and insulation on a silicon substrate. Lab Chip 1, 143.

Jo, K., Dhingra, D. M., Odijk, T., De Pablo, J. J., Graham, M. D., Runnheim, R., Forrest, D., et Schwartz, D. C. (2007). A single-molecule barcoding system using nanoslits for DNA analysis. Proceedings of the National Academy of Sciences 104, 2673. 
Kaji, N., Tezuka, Y., Takamura, Y., Ueda, M., Nishimoto, T., Nakanishi, H., Horiike, Y., et Baba, Y. (2004). Separation of long DNA molecules by quartz nanopillar chips under a direct current electric field. Anal. Chem 76, 15-22.

Mannion, J. T., Reccius, C. H., Cross, J. D., et Craighead, H. G. (2006). Conformational analysis of single DNA molecules undergoing entropically induced motion in nanochannels. Biophysical journal 90, 4538-4545.

Mawatari, K., Tsukahara, T., Sugii, Y., et Kitamori, T. (2010). Extended-nano fluidic systems for analytical and chemical technologies. Nanoscale 2, 1588.

Odom, T. W., Thalladi, V. R., Love, J. C., et Whitesides, G. M. (2002). Generation of 30- 50 nm Structures Using Easily Fabricated, Composite PDMS Masks. J. Am. Chem. Soc $124,12112-12113$.

Ou, J., Cho, J., Olson, D., et Dorfman, K. (2009). DNA electrophoresis in a sparse ordered post array. Phys. Rev. E 79. Available at: http://link.aps.org/doi/10.1103/PhysRevE.79.061904.

Ou, J., Joswiak, M. N., Carpenter, S. J., et Dorfman, K. D. (2011). Plasma thinned nanopost arrays for DNA electrophoresis. J. Vac. Sci. Technol. A 29, 011025.

Park, S., Huh, Y. S., Craighead, H. G., et Erickson, D. (2009). A method for nanofluidic device prototyping using elastomeric collapse. Proceedings of the National Academy of Sciences 106, 15549.

Perkins, T., Smith, D., Larson, R., et Chu, S. (1995). Stretching of a single tethered polymer in a uniform flow. Science 268, 83-87.

Piruska, A., Gong, M., Sweedler, J. V., et Bohn, P. W. (2010). Nanofluidics in chemical analysis. Chem. Soc. Rev. 39, 1060.

Qiao, R. (2006). Effects of molecular level surface roughness on electroosmotic flow. Microfluid Nanofluid 3, 33-38.

Randall, G. C., et Doyle, P. S. (2006). Collision of a DNA Polymer with a Small Obstacle. Macromolecules 39, 7734-7745.

Reisner, W., Morton, K., Riehn, R., Wang, Y., Yu, Z., Rosen, M., Sturm, J., Chou, S., Frey, E., et Austin, R. (2005). Statics and Dynamics of Single DNA Molecules Confined in Nanochannels. Phys. Rev. Lett. 94. Available at: http://link.aps.org/doi/10.1103/PhysRevLett.94.196101.

Reyes, D. R., Iossifidis, D., Auroux, P.-A., et Manz, A. (2002). Micro Total Analysis Systems. 1. Introduction, Theory, and Technology. Anal. Chem. 74, 2623-2636.

Rogers, J. A., Paul, K. E., Jackman, R. J., et Whitesides, G. M. (1998). Generating 90 nanometer features using near-field contact-mode photolithography with an elastomeric phase mask. Journal of Vacuum Science \& Technology B: Microelectronics and Nanometer Structures 16, 59. 
Rogers, J. A., Paul, K. E., Jackman, R. J., et Whitesides, G. M. (1997). Using an elastomeric phase mask for sub-100 nm photolithography in the optical near field. Applied Physics Letters 70, 2658.

Sanders, G., H.W., et Manz, A. (2000). Chip-based microsystems for genomic andproteomic analysis. Trends in Analytical Chemistry 19, 364-378.

Stellwagen, N. C., Gelfi, C., et Righetti, P. G. (1997). The Free Solution Mobility of DNA. Biopolymers 42, 687-703.

Tang, K. C., Liao, E., Ong, W. L., Wong, J. D. S., Agarwal, A., Nagarajan, R., et Yobas, L. (2006). Evaluation of bonding between oxygen plasma traeted polydimethyl siloxane and passivated silicon. Journal of micromechanics and microengineering 16, 155-161.

Tegenfeldt, J. O., Prinz, C., Huang, R. L., Austin, R. H., Chou, S. Y., Cox, E. C., Sturm, J. C., et Cao, H. (2004). Micro- and nanofluidics for DNA analysis. Analytical and Bioanalytical Chemistry 378, 1678-1692.

Viovy, J. L. (2000). Electrophoresis of DNA and other polyelectrolytes: Physical mechanisms. Reviews of Modern Physics 72, 813.

Whitesides, G. M. (2011). What Comes Next? Lab Chip 11, 191.

Zhou, W., Huang, Y., Menard, E., Aluru, N. R., Rogers, J. A., et Alleyne, A. G. (2005). Mechanism for stamp collapse in soft lithography. Appl. Phys. Lett. 87, 251925. 
Figure 1: Principle and Results of PDMS-based Phase Shift Lithography

(A) This figure sketches the principle of PPSL, highlighting the dimensions of the PDMS phase mask as well as the photoresist thickness. (B) The upper panels show scanning electron micrographs of $200 \mathrm{~nm}$ and $100 \mathrm{~nm}$ PPSL AZ 1505 linear features on silicon wafers. The lower panels represent scanning electron micrographs of $200 \mathrm{~nm}$ punctual patterns of AZ1505 with cylindrical and ovoid geometries, which are obtained by two consecutive exposures with two different angular shifts $\left(90^{\circ}\right.$ and $30^{\circ}$, respectively). Scale bars $=2 \mu \mathrm{m}$, unless mentioned.

\section{Figure 2: Integration of PPSL features in functional nanofluidic systems}

(A) An artistic view of a device composed of nanopost arrays is represented in the left panel. Nanopost arrays of different geometries are observed by electron microscopy (right panel), and the insets show tilted zooms of single elliptical (left) and cylindrical (right) nanopost, which are $800 \mathrm{~nm}$ in height. Note that the boundaries of the channels were defined using a rectangular canvas made out of an aluminum foil that was deposited on top of the phase shift mask. (B) The left panel depicts the geometry of a silicon nanochannels device, and the inset specifies its dimensions. The right panel is a scanning electron micrograph of the functional system. Note that nanochannels are first etched on flat silicon wafers, followed by microfluidic channels etching. (C) The mold for the fabrication of h-PDMS nanochannels is schematized in the left panel, and imaged by scanning electron microscopy in the right panel. The width as well as the height of these nanochannels is $200 \mathrm{~nm}$. (D) SEM of h-PDMS nanochannel chip, highlighting the sharp interface between microchannels (on the left) and nanochannels (on the right). Note that a $5 \mathrm{~nm}$ gold layer was deposited on PDMS to improve imaging conditions. Scale bars $=2 \mu \mathrm{m}$. Note that micropillars were engraved in microchannels in order to facilitate the uptake of DNA molecules in nanochannels by unraveling their natural coiled conformation (cylindrical patterns in the micrographs of (B), (C), and (D)).

\section{Figure 3: Single DNA molecule manipulation in PPSL nanofluidic systems}

(A) The plot represents the temporal evolution of the center of mass of a $\lambda$-DNA molecule moving at a speed of $24 \mu \mathrm{m} / \mathrm{s}$ and colliding with a $140 \mathrm{~nm}$ nanopost. The upper fluorescence micrographs show the molecule conformation in the course of the collision. The picture in the inset is the camera full-frame, showing multiple DNA collisions and the absence of clogging. (B) The plot shows the length of a $\lambda$-DNA over time, as the molecule enters inside a nanochannel with selected snapshots of the relaxation in the upper panel. The full-frame of the camera in the inset shows the exquisite signal to noise ratio, which is characterized by a signal to noise ratio of 15+/-3 (signal divided by standard deviation of the background noise) for inter-frame intervals of $30 \mathrm{~ms}$. The histogram in the inset represents the steady-state $\lambda$ DNA size distribution in nanochannels $(0.5 X$ TBE, $n=30)$. Scale bars $=10 \mu \mathrm{m}$. Red arrows indicate the direction of DNA migration.

\section{Figure 4: DNA electrophoresis in h-PDMS nanochannels}

(A) The graphs represents the migration speed of $\lambda$-DNA for different electric fields inside 70 $\mu \mathrm{mx} 2 \mu \mathrm{m}$ microchannels (left panel), and $200 \mathrm{nmx} 200 \mathrm{~nm}$ nanochannels etched in PDMS or in silicon (blue and red datasets, respectively, in the right panel). Note that we systematically 
assessed the micro- vs. nano-fluidic response in the same chip. Also, the actuating field is stronger in nanochannels because the insertion of molecules in nanochannels has to be triggered by higher electric fields. (B) The array summarizes the mobility of $\lambda$-DNA (units $=$ $10^{-4} \mathrm{~cm}^{2} / \mathrm{Vs}$ ) inside micro- and nano-channels in different buffer conditions. Note that our estimates of the mobility were stable over periods of at least 2 hours. 


\section{Supplementary Information}

\section{Figure S1: Nanoposts size distribution}

The plot shows the size distribution of silicon cylindrical nanopost obtained by image analysis using the "fit ellipse" function in ImageJ of magnified SEM views of individual nanoposts, as shown in the insets of Fig. 2A. Gaussian fit gives a mean diameter of $268.3 \mathrm{~nm}$, with a standard deviation of $7.5 \mathrm{~nm}$, that is less than $3 \%$.

Figure S2: Resolution loss at the interface micro/nanochannels for $2 \mu \mathrm{m}$ thick microchannels

SEM of h-PDMS nanochannel chips with $2 \mu \mathrm{m}$ thick microchannels, which are located on the left of the SEM image. The lateral dimension of nanochannels is $\sim 700 \mathrm{~nm}$ at their entrance, and it then reduces to reach $200 \mathrm{~nm} \sim 15 \mu \mathrm{m}$ apart from the entrance. Scale bar $=5 \mu \mathrm{m}$. 
Fig. 1
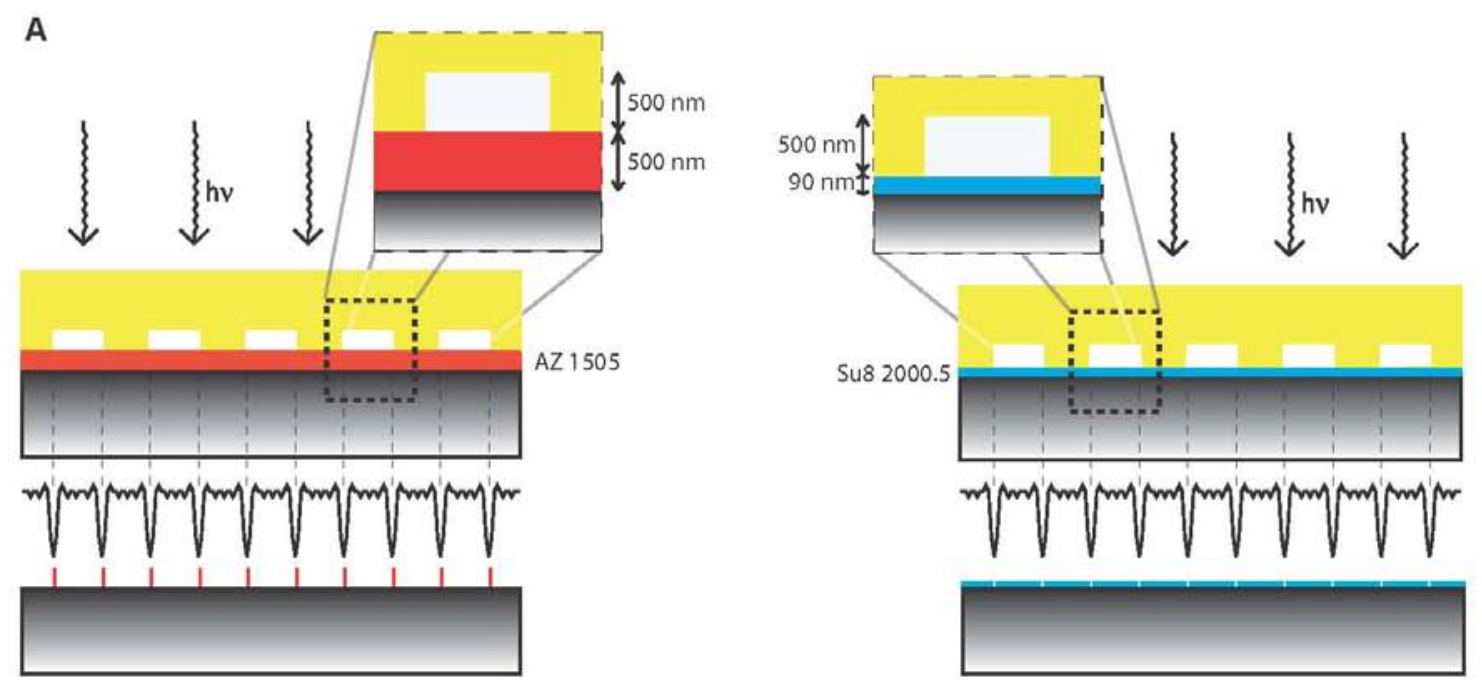

B
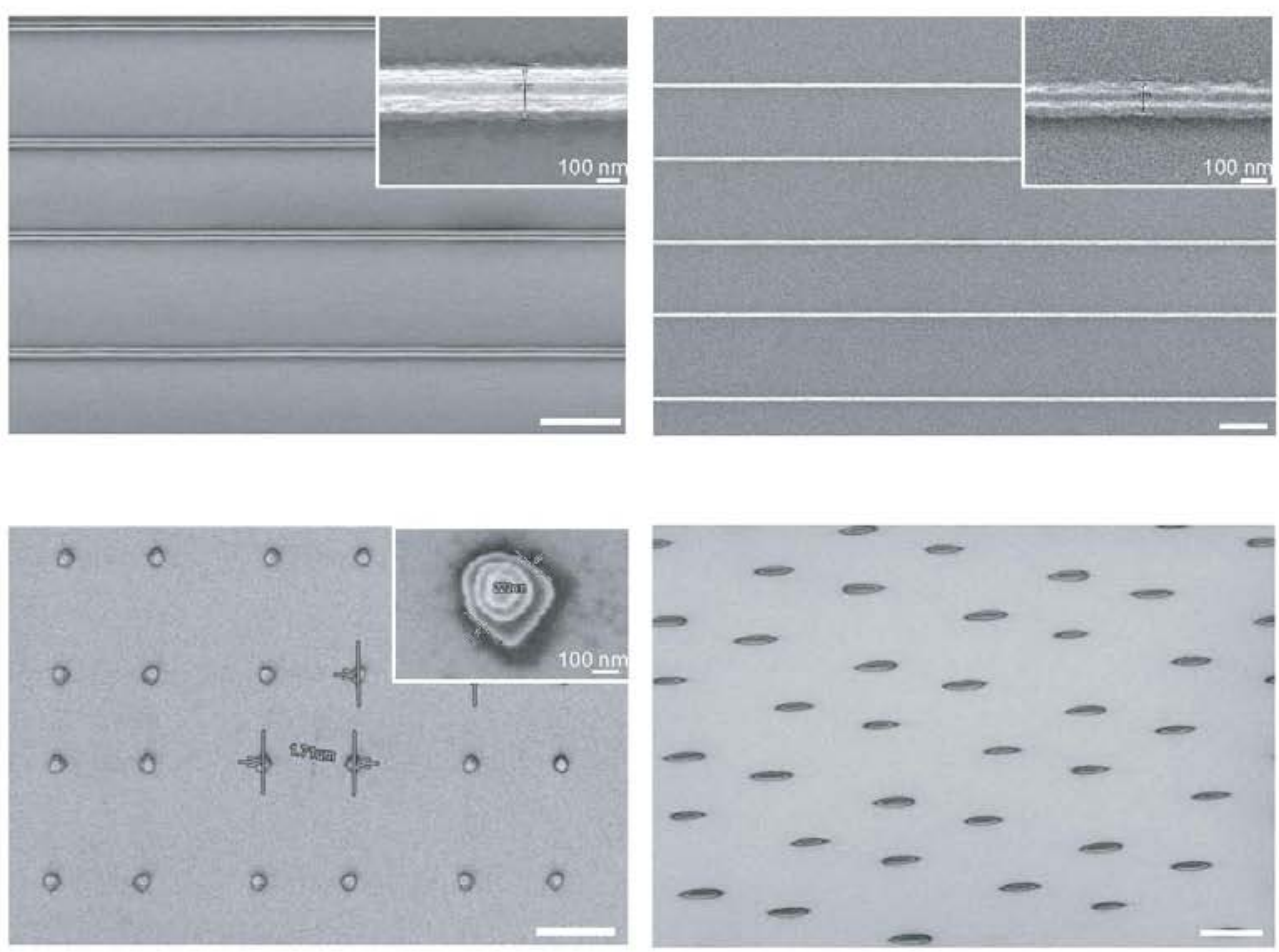
Fig. 2

A
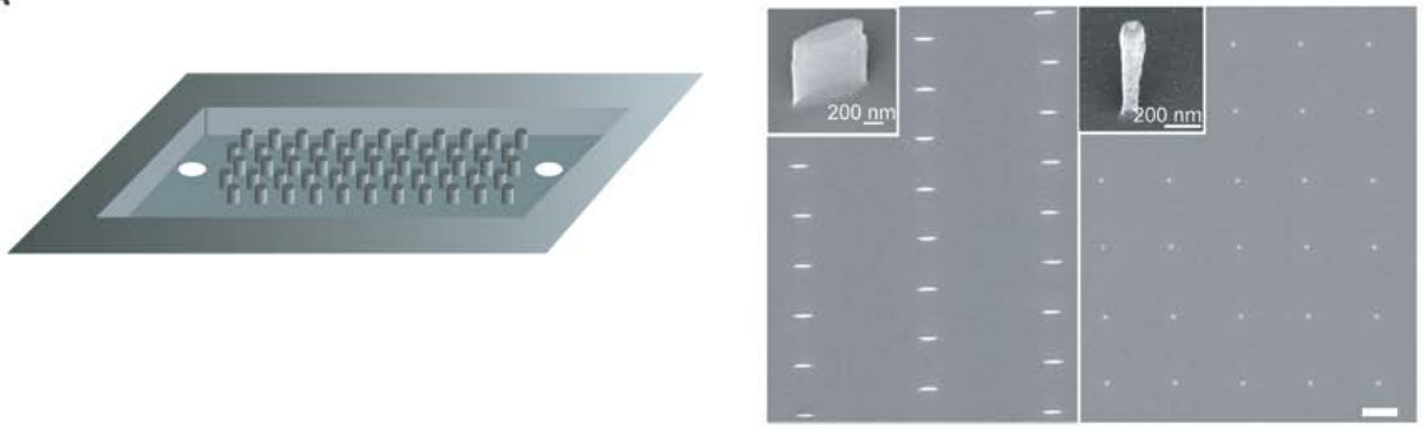

B
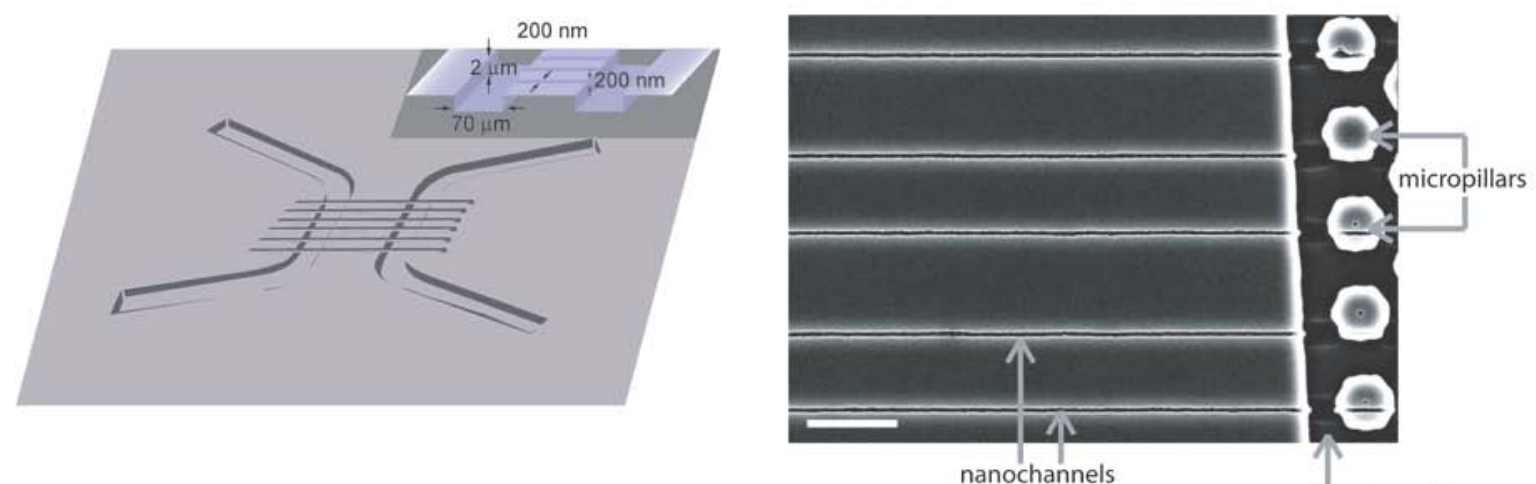

C
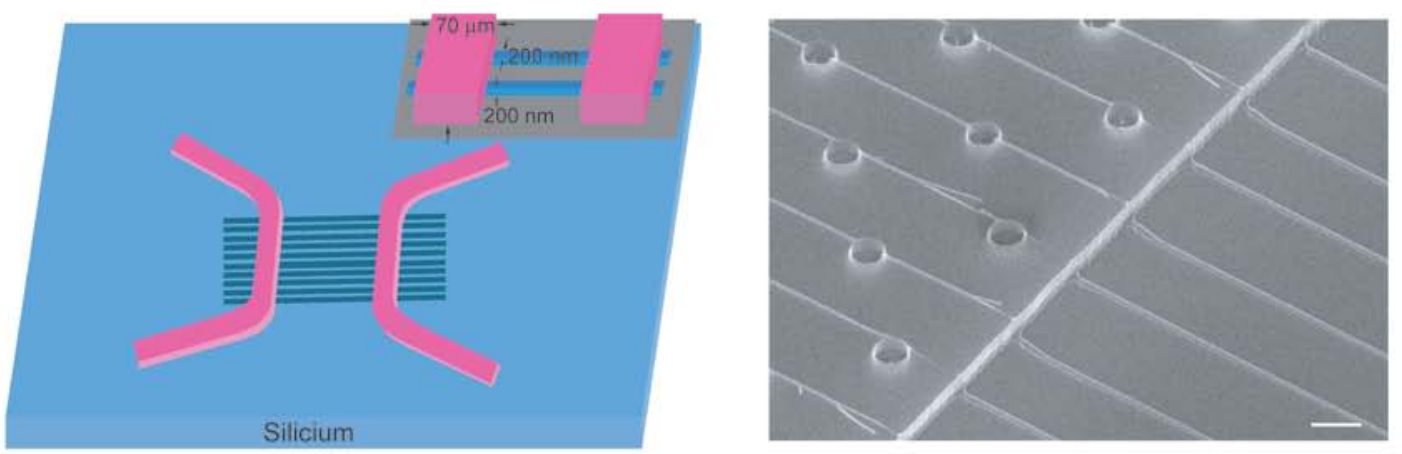

D

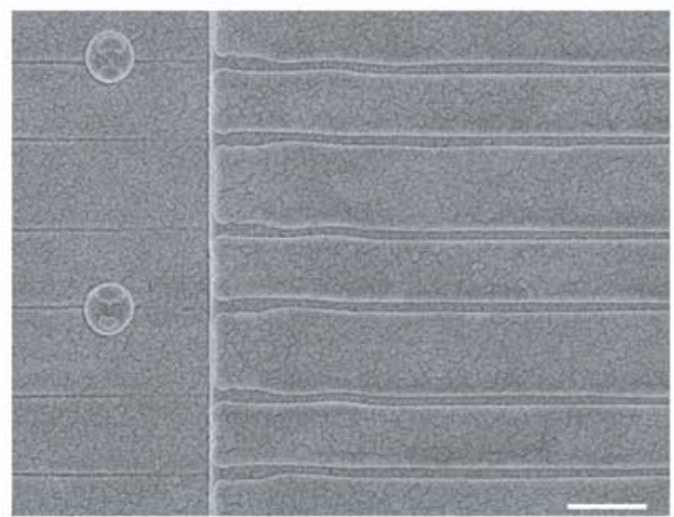


Fig. 3

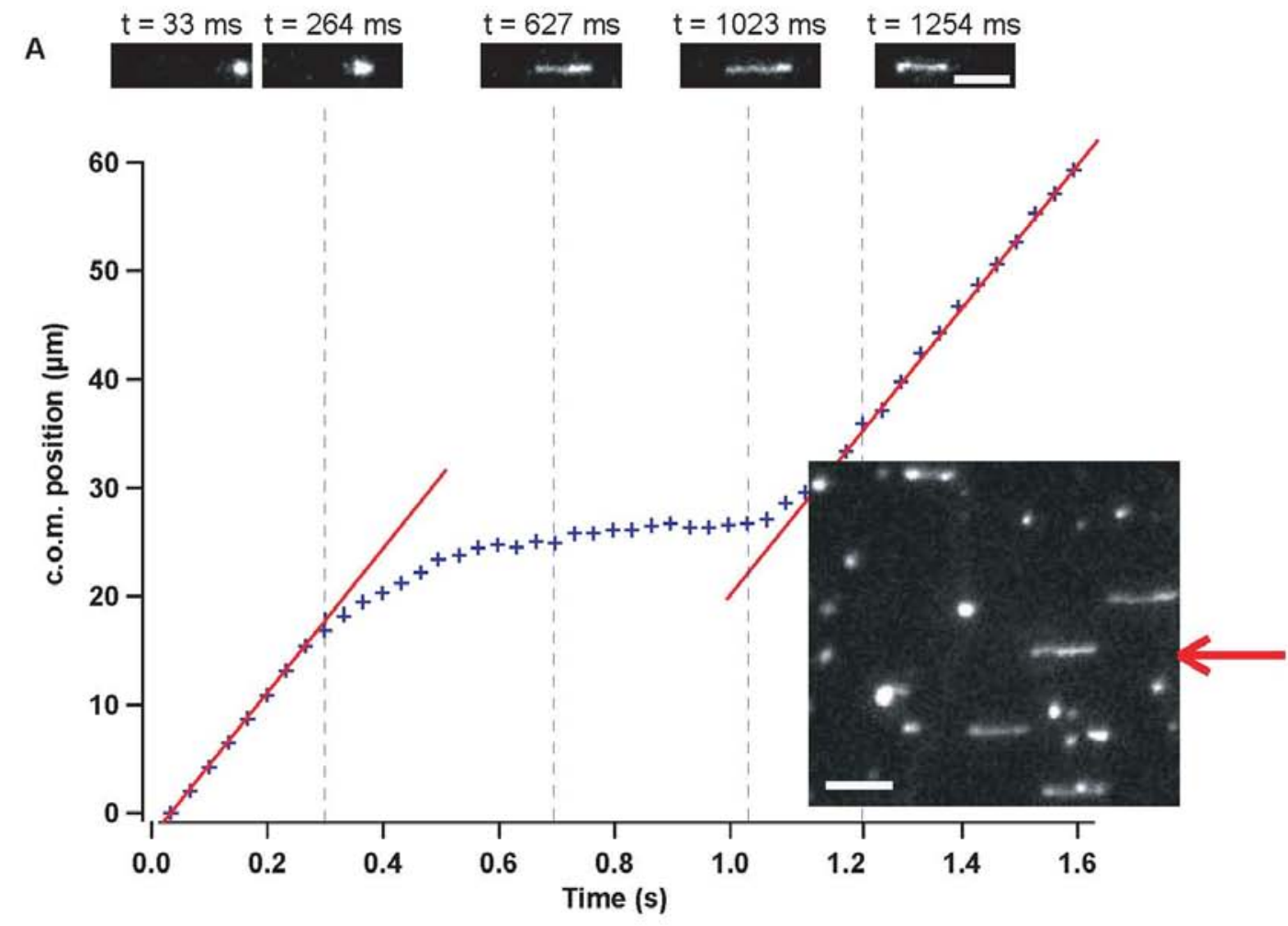

B

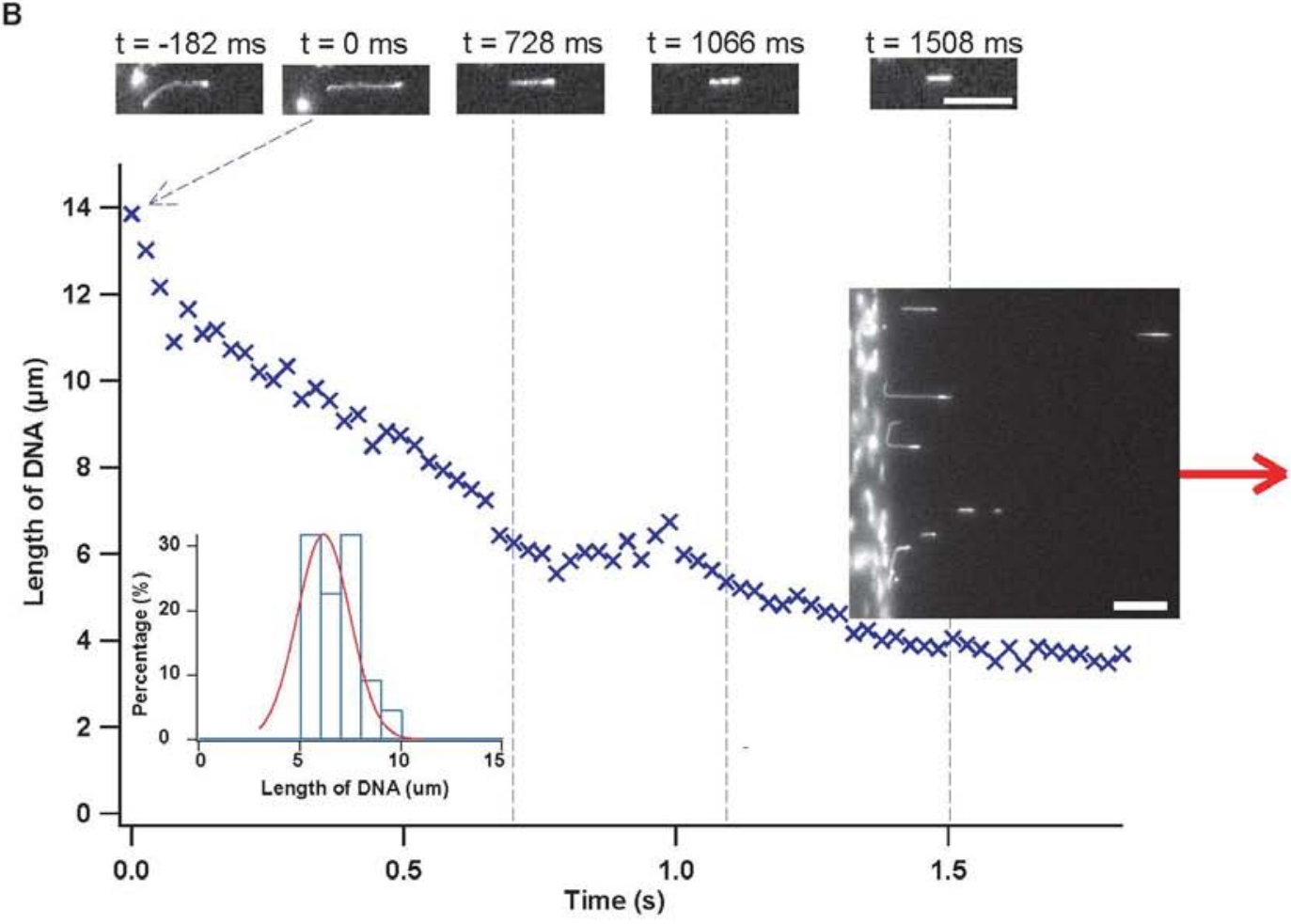


Fig.4
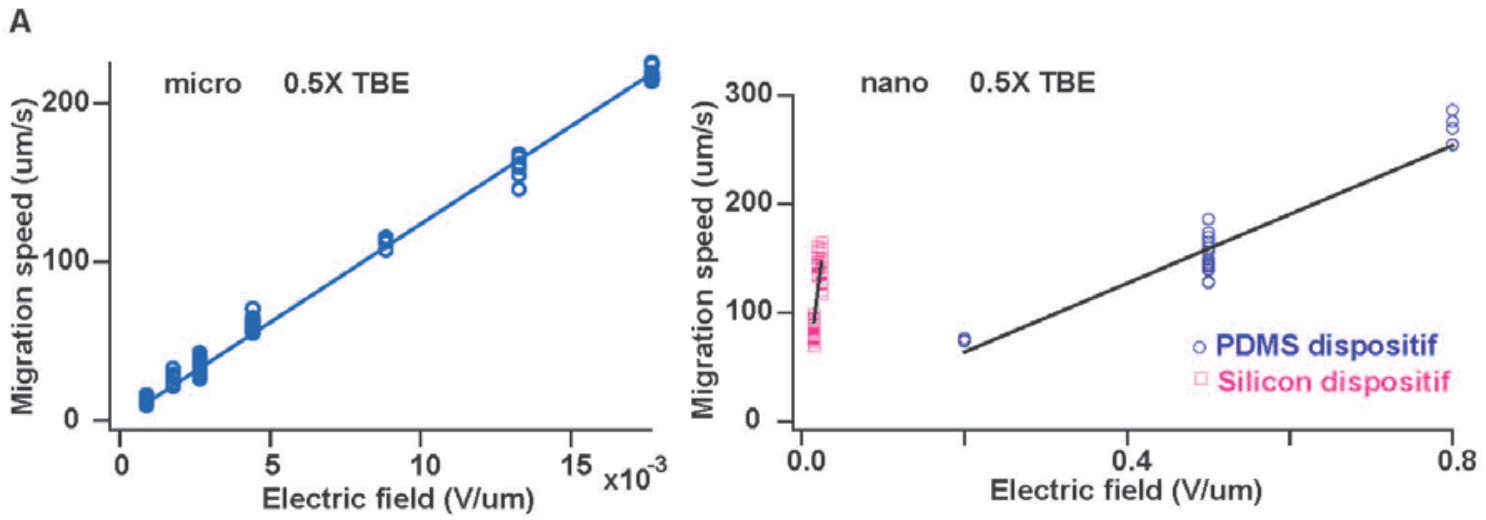

B

\begin{tabular}{|l|c|r|}
\hline \multicolumn{1}{|c|}{ Condition } & micro & nano \\
\hline $0.05 x$ TBE & 2.5 & 0.13 \\
$0.1 \times$ TBE & 2.8 & 0.09 \\
$0.5 x$ TBE & 3.2 & 0.17 \\
1x TBE & 2.4 & 0.14 \\
$2 \times$ TBE & 2.1 & 0.21 \\
phosphate $\mathrm{pH}=5.3$ & 2.2 & 0.22 \\
phosphate $\mathrm{pH}=7.2$ & 2.2 & 0.24 \\
phosphate $\mathrm{pH}=8.6$ & 2.4 & 0.13 \\
super saturated. 0.5x TBE & 2.8 & 0.12 \\
rinsed with EDTA. 0.5x TBE & 5.1 & 0.03 \\
100nm fluo particles. 0.5x TBE & 1.6 & 0.03 \\
0.5x TBE in silicon dispositif & 2.1 & 1.09 \\
\hline
\end{tabular}


Fig. S1

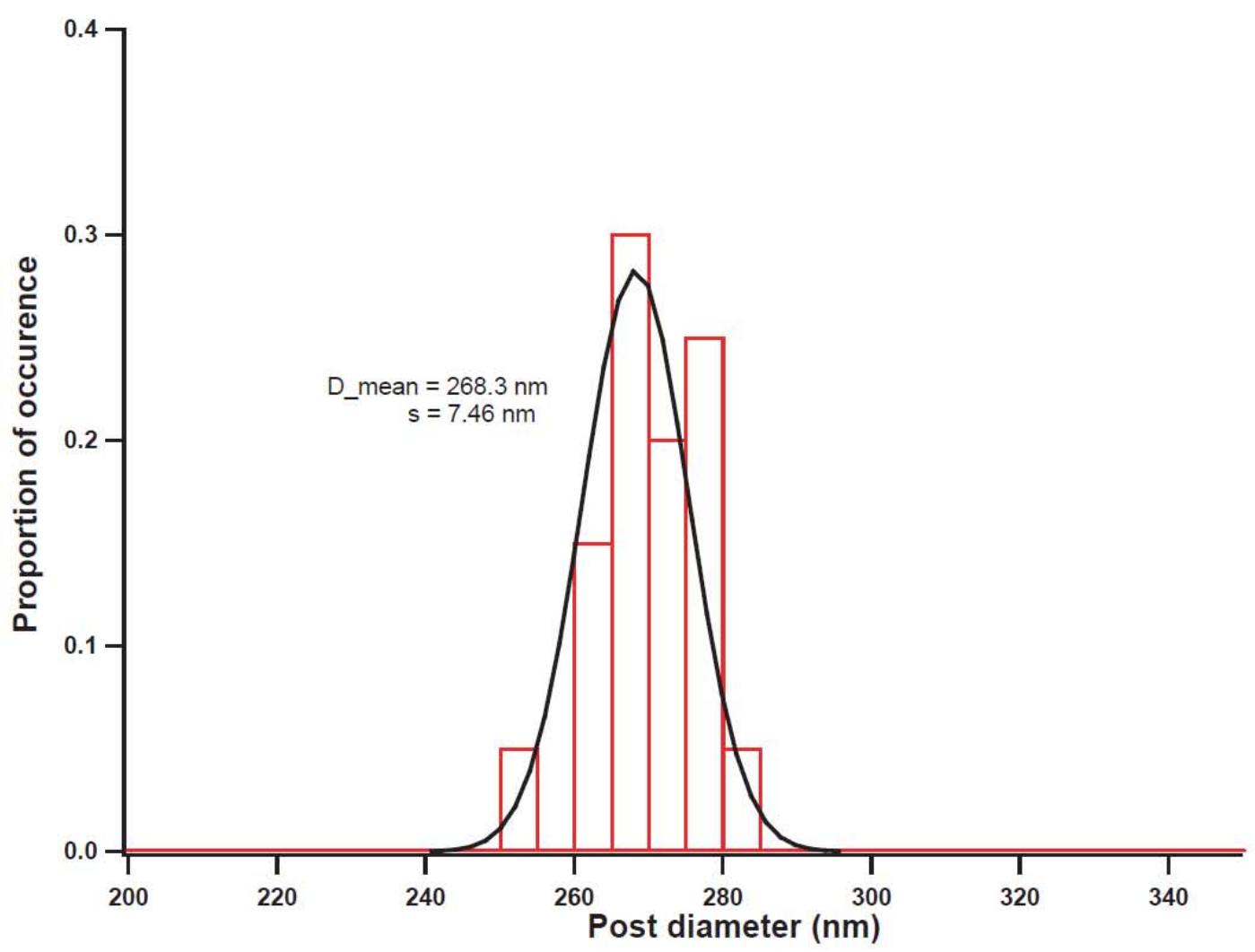

Fig. 52

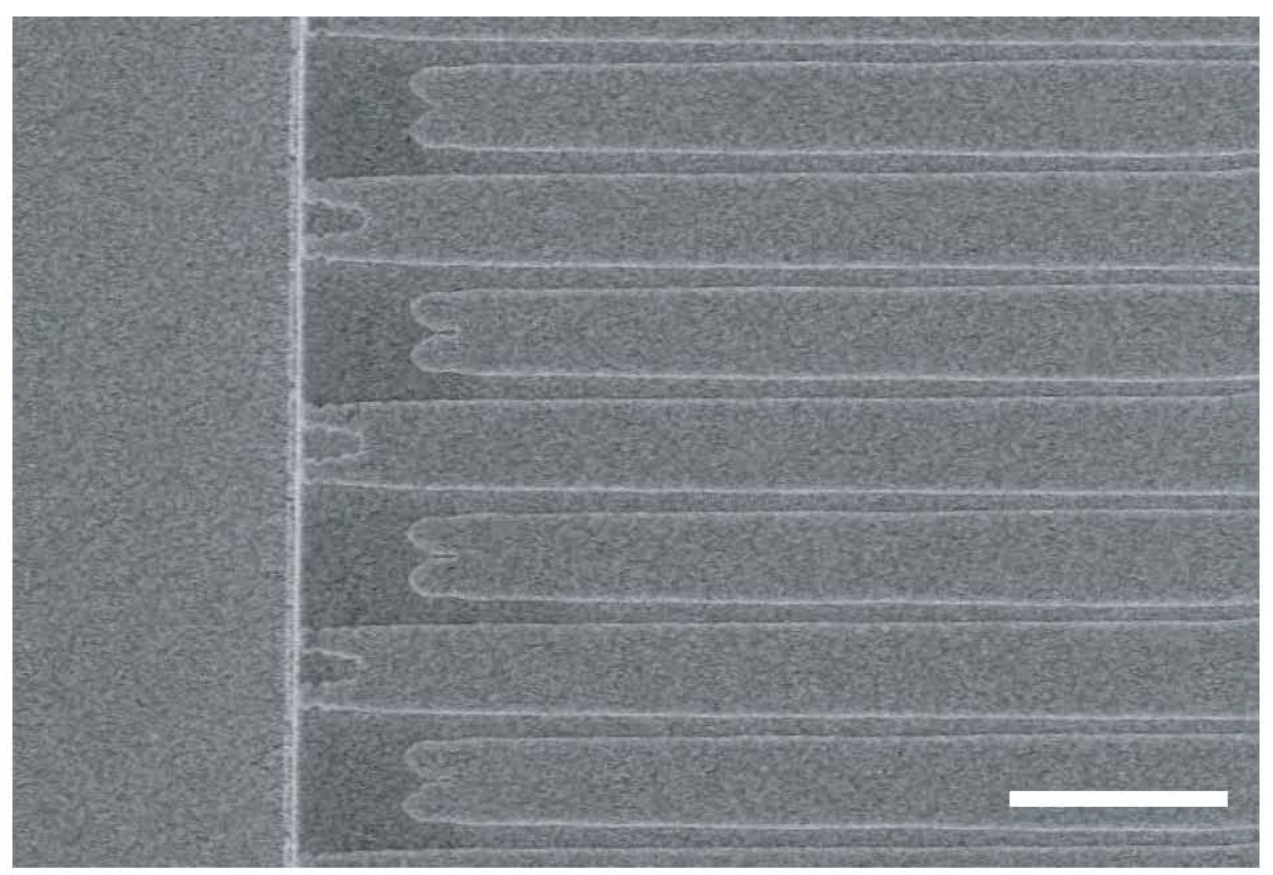

